\title{
Review Article \\ Emerging Roles of MicroRNAs in EGFR-Targeted Therapies for Lung Cancer
}

\author{
Fei Han, ${ }^{1}$ Jinxi He, ${ }^{2}$ Feng Li, ${ }^{3}$ Jiali Yang, ${ }^{3}$ Jun Wei, ${ }^{1,3}$ William C. Cho, ${ }^{4}$ and Xiaoming Liu ${ }^{1,3,5}$ \\ ${ }^{1}$ Human Stem Cell Institute of the General Hospital, Ningxia Medical University, Yinchuan, Ningxia 750004, China \\ ${ }^{2}$ Department of Thoracic Surgery of the General Hospital, Ningxia Medical University, Yinchuan, Ningxia 750004, China \\ ${ }^{3}$ Center of Laboratory Medicine of the General Hospital, Ningxia Medical University, Yinchuan, Ningxia 750004, China \\ ${ }^{4}$ Department of Clinical Oncology, Queen Elizabeth Hospital, Kowloon, Hong Kong \\ ${ }^{5}$ Key Laboratory of Ministry of Education for Conservation and Utilization of Special Biological Resources in Western China, \\ Ningxia University, Yinchuan, Ningxia 750021, China
}

Correspondence should be addressed to William C. Cho; williamcscho@gmail.com and Xiaoming Liu; liuxiaoming@nxmu.edu.cn

Received 10 February 2015; Accepted 20 May 2015

Academic Editor: Martin Sebastian Staege

Copyright @ 2015 Fei Han et al. This is an open access article distributed under the Creative Commons Attribution License, which permits unrestricted use, distribution, and reproduction in any medium, provided the original work is properly cited.

\begin{abstract}
Lung cancer is a leading cause of cancer mortality worldwide. Several molecular pathways underlying mechanisms of this disease have been partly elucidated, among which the epidermal growth factor receptor (EGFR) pathway is one of the well-known signaling cascades that plays a critical role in tumorigenesis. Dysregulation of the EGFR signaling is frequently found in lung cancer. The strategies to effectively inhibit EGFR signaling pathway have been mounted for developing anticancer therapeutic agents. However, most anti-EGFR-targeted agents fail to repress cancer progression because of developing drug-resistance. Therefore, studies of the mechanisms underpinning the resistance toward anti-EGFR agents may provide important findings for lung cancer treatment using anti-EGFR therapies. Recently, increasing numbers of miRNAs are correlated with the drug resistance of lung cancer cells to antiEGFR agents, indicating that miRNAs may serve as novel targets and/or promising predictive biomarkers for anti-EGFR therapy. In this paper, we summarize the emerging role of miRNAs as regulators to modulate the EGFR signaling and the resistance of lung cancer cells to anti-EGFR therapy. We also highlight the evidence supporting the use of miRNAs as biomarkers for response to anti-EGFR agents and as novel therapeutic targets to circumvent the resistance of lung cancer cells to EGFR inhibitors.
\end{abstract}

\section{Introduction}

Lung cancer is a heterogeneous disease, which is the leading cause of cancer-related mortality worldwide. It can be broadly classified into non-small-cell lung cancer (NSCLC) and small cell lung cancer based on clinical, histological, molecular, and endocrinological characteristics. Using histological features, NSCLC can be further subdivided into large-cell carcinoma, bronchoalveolar lung cancer, adenocarcinoma, squamous carcinoma, and mixed histological types (e.g., adenosquamous carcinoma) [1,2]. NSCLC accounts for more than $85 \%$ of all patients with lung cancer. To date, platinum-based doublets remain the mainstay in the treatment of patients with advanced NSCLC [2]. With an increased understanding of the mechanisms underpinning lung cancer development and progression, a number of novel agents specifically targeting oncogenic pathways have been developed and applied to treat lung cancer [3], among which the inhibitor targeting epidermal growth factor receptor (EGFR) signaling cascades is one of the most broadly used agents implemented in clinical practice [3-5].

EGFR is a receptor of tyrosine kinase (RTK), which consists of an $\mathrm{N}$-terminus extracellular ligand-binding site, a hydrophobic transmembrane domain, and a C-terminus intracellular region with tyrosine kinase activity [5]. The EGFR signaling network plays a central role in the growth and maintenance of epithelial tissues. The dysregulation and/or hyperactivation of the EGFR signaling pathway are frequently found in epithelial lung tumor entities, in which the hyperactivated EGFR signaling is associated with advanced lung 
cancer and poor prognosis [6]. Therefore, EGFR and its downstream signaling components can be used as major targets in developing novel agents for cancer treatment, such as chimeric monoclonal antibodies (cetuximab and panitumumab) [2] and tyrosine kinase inhibitors (TKIs) (gefitinib, erlotinib, and afatinib) [7-9]. However, the clinical benefits of these anti-EGFR agents are often limited, mainly due to the heterogeneity of lung cancer and the drug resistance to anti-EGFR therapy [10]. Consequentially, a large number of studies focus on the mechanisms underpinning the resistance toward anti-EGFR agents.

MicroRNAs (miRNAs) are a class of small noncoding RNAs that act as key posttranscriptional regulators of gene expression. They can functionally impact cell fate determination by regulating the expression of critical proteins; thus miRNAs play a pivotal role in the diverse processes of human cancer, acting as either tumor suppressors or oncogenes [11-13]. Recently, several studies have demonstrated that EGFR is a target of a number of miRNAs, and vice versa a mutation or activity of the EGFR signaling pathway can alter the expressions of miRNAs in lung cancer (Table 1) [14-17]. The involvement of miRNAs in the EGFR signaling pathway of lung cancer development and target therapy has recently gained increasing attentions [16]. In this review, we summarize the emerging role of miRNAs as modulators to regulate the EGFR signaling pathway and mainly focus on miRNAs as predictive biomarkers for anti-EGFR therapy and as novel targets to reverse the resistance of lung cancer cells to EGFR inhibitors.

\section{2. miRNAs Target EGFR in Human Lung Cancers}

miRNAs have gained increasing interest owing to their widespread occurrence and diverse functions as regulatory molecules in many signaling pathways, including the EGFR signaling pathway. Accumulating evidence has revealed that miRNAs are oncomirs or tumor suppressors by targeting the EGFR signaling pathway in different types of cancer. Table 1 lists several miRNAs that regulate the EGFR signaling pathway in lung cancer and Figure 1 shows the miRNAs that target the EGFR signaling pathway.

Using bioinformatics tools, Chan et al. predicted $138 \mathrm{miR}-$ NAs that potentially target EGFR in NSCLC, some of these miRNAs were confirmed experimentally [18]. Among these miRNAs, miR-7 has been demonstrated to downregulate EGFR in different cancer cells. Mechanistically, miR-7 could directly target EGFR by binding three seeding sequences in human EGFR mRNA $3{ }^{\prime}$-UTR $[19,20]$. Apart from its ability to directly target EGFR mRNA, miR-7 can also target several downstream effectors of the EGFR signaling pathway, including the proto-oncogene serine/threonine protein kinase RAF-1, protein kinase B Akt, and extracellular signalregulated kinase 1/2 (ERK1/2), sequentially inhibits cancer cell migration, invasion, and metastasis [16, 20]. In lung cancer, miR-7 was upregulated in $60 \%$ of NSCLC fineneedle aspirates, which could be induced by both wild type and mutant EGFR L858R, and plays an oncogenic role by activating the rat sarcoma viral oncogene homolog
(RAS)/ERK/c-Myc signaling axis to promote lung carcinogenesis by repressing the transcriptional regulator Ets 2 transcriptional repression factor (ERF) $[16,21]$. In this context, an activation of EGFR or ectopic expression of RAS and c-Myc could induce miR-7 transcription in an extracellular signalregulated kinase- (ERK-) dependent manner. This notion was supported by findings of that c-Myc bound to the miR-7 promoter and enhanced its activity, and an enforced expression of miR-7 promoted cell growth and tumor formation in lung cancer cells and significantly increased the mortality of nude mice orthotopically implanted with lung cancers. Molecular analysis further revealed that miR-7 could directly target ERF, a seeding sequence of miR-7 that was confirmed in the coding sequence of ERF, suggesting that miR-7 may act as an important regulator of EGFR-mediated oncogenesis and can be served as a novel prognostic biomarker and therapeutic target in lung cancer [21].

miRNA profiling of lung cancer cell lines and lung tissues has demonstrated that miRNAs are emerging as unique effectors of the EGFR signaling pathway in lung cancer, in which miRNAs are correlated with the expression of EGFR and/or the EGFR mutant status or signaling activities [22, 23]. Analyzing miRNA expression profiling of lung cancer, Dacic et al. observed a correlation of miRNAs with mutational status of EGFR in lung adenocarcinomas, in which miR155 was upregulated only in EGFR/KRAS-negative samples, and miR-25 was upregulated only in EGFR-positive group and miR-495 was upregulated only in KRAS-positive adenocarcinomas. Conversely, let-7g was dramatically downregulated in EGFR/KRAS negative adenocarcinomas [23]. Such a correlation was also found in other mRNAs. For examples, miR-542-5p could downregulate EGFR mRNA and protein expression in human lung cancer H3255, A549, and HCC827 cells and inhibit the growth of these cancer cells. Interestingly, an inverse correlation of miR-542-5p transcript and EGFR protein levels was found in human lung cancer tissues [24]. Such an inverse correlation of miRNA expression and the EGFR signaling pathway was also found in miR-133a whose expression was negatively correlated with cell invasiveness in lung cancer cell lines, by targeting insulin-like growth factor 1 receptor (IGF-1R), TGF-beta receptor type-1 (TGF $\beta$ R1), and EGFR [25]. Similarly, Chan et al. also demonstrated that miR-146a inhibited cell growth and induced cell apoptosis by suppressing the EGFR downstream signaling components and the migratory capacity in various NSCLC cell lines (H358, H1650, H1975, HCC827, and H292), through an EGFR mutation status independent mechanism of directly targeting the EGFR and nuclear factor kappa beta (NF- $\kappa \mathrm{B})$ signaling pathways [18].

In line with the regulatory role of miRNAs in the EGFR signaling pathway, the aberrant expression and/or mutation(s) of EGFR may also alter the expression of miRNAs in lung cancer. For instance, the expression of some miRNAs, such as miR-21 was altered more remarkably in a lung cancer with EGFR mutations relative to those without these mutations [26], suggesting that the EGFR signaling pathway is not only regulated by tumor-suppressive miRNAs, but also has potential to regulate some miRNAs acting as oncogene. In a recent study by Guo et al., the authors found that aberrant 


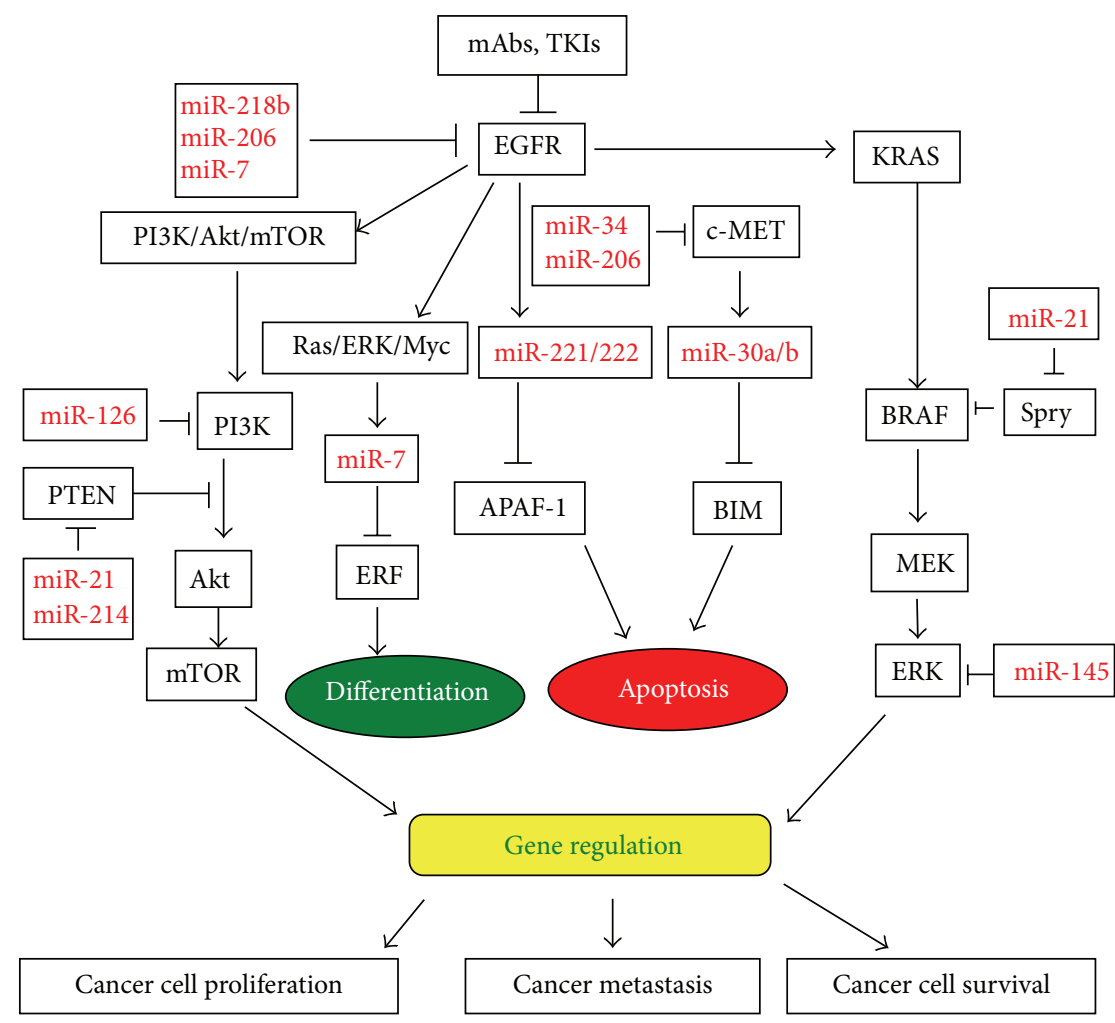

FIGURE 1: An illustration representing microRNAs (miRNAs) and their targets involved in EGFR signaling pathway in lung cancer and antiEGFR therapy. The depicted miRNAs target important signaling pathways in lung cancer development and resistance to anti-EGFR agents.

TABLE 1: MicroRNAs that target EGFR signaling pathway involved in lung cancer.

\begin{tabular}{|c|c|c|c|}
\hline MicroRNA(s) & Regulation & Potential function(s) & Reference(s) \\
\hline let-7g & Down & Dramatically downregulated in EGFR/KRAS negative lung adenocarcinomas & [23] \\
\hline miR-7 & Down & $\begin{array}{l}\text { Inhibits EGFR-PI3K-AKT signaling and reverses radio-resistance in various } \\
\text { cancer cells }\end{array}$ & {$[20]$} \\
\hline miR-27a & Down & Directly targets EGFR and contributes to mutant p53 gain-of-function & {$[36,37]$} \\
\hline miR-34a & Down & Regulates Axl receptor tyrosine kinase by targeting SIRT1 and MEK1 & [38] \\
\hline $\mathrm{miR}-128 \mathrm{~b}$ & Down & Directly regulates EGFR expression in NSCLC & {$[15,18]$} \\
\hline miR-133a & Down & Repress EGFR signaling by directly targeting IGF-1R, TGF $\beta$ R1, and EGFR & [25] \\
\hline miR-133b & Down & $\begin{array}{l}\text { Suppresses EGFR pathway signaling and enhances susceptibility to EGFR-TKI } \\
\text { in lung cancer cells by directly targeting EGFR }\end{array}$ & [39] \\
\hline miR-145 & Down & Negatively regulates EGFR expression in lung cancer cells & {$[31]$} \\
\hline miR-146a & Down & Inhibit EGFR in NSCLC cancer cells & {$[40,41]$} \\
\hline miR-146b-5p & Down & Suppressed EGFR expression in glioblastoma cell lines & {$[42]$} \\
\hline miR-200 & Down & $\begin{array}{l}\text { Regulates EMT in anaplastic thyroid cancer cells and bladder cancer cells and } \\
\text { reverses resistance of EGFR therapy }\end{array}$ & {$[43,44]$} \\
\hline miR-206 & Down & $\begin{array}{l}\text { Suppresses EGFR signaling in squamous lung cancer cells by directly targeting } \\
\text { EGFR and MET }\end{array}$ & {$[45]$} \\
\hline $\begin{array}{l}\operatorname{miR}-542-5 p \\
1203,1237,541 \\
1911\end{array}$ & Down & Downregulates EGFR in human lung cancer cells & {$[20,24]$} \\
\hline $\operatorname{miR}-21$ & Up & Regulate the EGFR/AKT pathway in a PTEN independent manner & {$[46]$} \\
\hline $\operatorname{miR}-24$ & Up & Activates EGFR signaling by targeting PTPN9 and PTPRF & {$[47]$} \\
\hline $\operatorname{miR}-25$ & Up & Upregulated in EGFR positive lung cancer & {$[23]$} \\
\hline miR-214 & Up & $\begin{array}{l}\text { Regulate acquired resistance to EGFR-TKIs in cancer cells through a } \\
\text { PTEN/AKT signaling pathway }\end{array}$ & {$[48]$} \\
\hline
\end{tabular}


activation of the EGFR signaling pathway downregulated miR-145 expression in NSCLC, an addition of EGFR inhibitor AG1478 that could restore the expression of miR-145 in lung cancer cells [27]. Using an Agilent microarray, Bjaanaes et al. examined the expression of miRNAs in 154 surgically resected lung adenocarcinomas and 20 corresponding normal lung tissue samples; they found that 129 miRNAs were strikingly differentially expressed in lung adenocarcinomas in comparison with normal lung tissues, among which 17 miRNAs were differentially expressed between tumors with EGFR-mutation and wild-type [22]. These studies imply a feedback regulatory mechanism between the EGFR signaling pathway and miRNAs in the development and progression of lung cancers.

\section{3. miRNAs Alter EGFR-TKI Responses in Lung Cancer}

Targeting therapy to the EGFR signaling pathway leads to development of EGFR tyrosine kinase inhibitors (TKIs), namely, gefitinib, erlotinib, and afatinib, for the treatment of patients with NSCLC who have EGFR mutations. Different from wild-type EGFR, mutations of EGFR may confer hypersensitivity to TKIs in advanced NSCLC [28], since cells with mutant EGFR transduce survival signals but have no effect in proliferative signals [8]. However, the clinical benefit of TKIs was limited as patients eventually develop resistance to these agents. $70 \%$ of this acquired resistance (AR) may be caused by a secondary mutation in the EGFR gene, such as T790M or amplification of the proto-oncogene hepatocyte growth factor receptor (c-MET). In gefitinib or erlotinib resistant tumor samples, about 50\% samples have been found to bear T790M mutation and the other $20 \%$ cases have cMET amplification. Mechanically, T790M can increase GTP affinity in the tyrosine kinase domain or block TKI binding to the tyrosine kinase domain of EGFR [29]. Other mechanisms, including the involvement of Anexelekto- (Axl-) kinase and a number of miRNAs in the AR of lung cancer to TKIs $[10,16]$, and several miRNAs have been demonstrated to be associated with EGFR mutations in lung cancer (Table 2) [30].

Increasing number of studies has revealed a correlation of the clinical responses to TKIs and the expressions of miRNAs. For example, loss of heterozygosity (LOH) at miR-128b is one of the most frequent genetic events in lung cancer. Weiss et al. found that $\mathrm{LOH}$ at miR-128b was frequent detected in lung cancer tissues and was positively correlated with clinical response and survival to gefitinib treatment [15]. Other studies showed that the restoration of miR-145 and miR-7 inhibited cancer cell growth in lung adenocarcinoma patients with EGFR activating mutation and could effectively target EGFR addicted and EGFR-TKI resistant tumors [21, 31]. In a recent study, Garofalo et al. demonstrated that RTK of EGFR and c-MET could induce miR-30b/30c/221/222 expressions, and an upregulation of miR-30b/30c/221/222 induced resistance to gefitinib in lung cancer cells by the regulation of BCL2-like 11 (BIM), phosphatase and tensin homolog (PTEN), and apoptotic peptidase activating factor 1 (APAF-1) expressions. In contrast, miR$103 / 203$ could induce gefitinib resistant cell apoptosis and promote mesenchymal to epithelial transformation (MET) by targeting protein kinase $\mathrm{C}$ varepsilon (PKC- $\varepsilon$ ) and sarcoma viral oncogene homolog (SRC), but the ectopic expressions of miR-30b/30c/221/222 conferred resistance to TKIs [32]. Such AR to TKIs could also be induced by miR-214 and miR-21 in lung cancer cells [33]. miR-214 expression was elevated in gefitinib resistant HCC827 lung cancer cells (HCC827/GR), which was inverse with PTEN expression. A knockdown of miR-214 in HCC827/GR showed a restoration of PTEN expression and resensitized HCC827/GR to gefitinib. Similar to miR-21, which was also more aberrantly expressed in EGFR-TKI-resistant lung cancer cell line PC9R relative to its parent cell PC9. The increased level of miR-21 was inversely correlated with the abundance of PTEN and programmed cell death protein 4 (PDCD4) proteins and positively correlated with the phosphatidylinositol-3-kinase (PI3K)/Akt signaling pathway. An inhibition of miR-21 induced apoptosis in PC9R cells and suppressed tumor growth in nude mice treated with EGFR-TKI. Clinically, circulating miR-21 level in EGFR-TKItreated NSCLC patients was significantly higher at the time of acquiring resistance over the baseline [26, 33]. Recent studies in lung adenocarcinoma of female nonsmokers also revealed that the expression of miR-183-3p, miR-195, and miR-122 was in plasma and associated with EGFR mutations in lung cancer $[34,35]$.

Intriguingly, several tumor-suppressive miRNAs exhibited an ability to enhance the cytotoxicity of EGFR-TKI to lung cancer cells, such as miR-126, miR-145, and miR146a [40, 54, 61]. Lung cancer cells H460 and A549 are significantly resistant to gefitinib, forced expression of miR126 and miR-145 showed an inhibited growth of cells and tumor xenografts and an enhanced cytotoxicity induced by gefitinib [54]. miR-146a is an extensively studied tumorsuppressive miRNA in many types of cancer, the polymorphism of the rs2910164CNG in pre-miR-146a was recently identified to be associated with the genetic susceptibility to lung cancer development in a Korean population [61]. In addition, a downregulation of miR-146a was reported in lung cancers, overexpression of miR-146a was found to suppress cell growth and migration, induce cellular apoptosis, and inhibit the EGFR downstream signaling components in lung cancer cell lines H358, H1650, H1975, HCC827, and H292. Importantly, forced expression of miR-146a could enhance the ability of EGFR-TKIs (gefitinib, erlotinib, and afatinib) and monoclonal antibody (cetuximab) to inhibit cell proliferation by targeting of the EGFR and NF- $\kappa \mathrm{B}$ signaling pathways [40].

The activation of c-MET is associated with both primary and acquired resistance to EGFR-TKIs in patients with NSCLCs [62]. Both EGFR and c-MET are RTKs that have been implicated in tumor progression as regulators of miRNA cluster $23 \mathrm{a} / 27 \mathrm{a} \sim 24-2$ in lung cancer, in which miR-27a can regulate both c-MET and EGFR [36]. Such a dual inhibitory role of miRNAs to the c-MET and EGFR oncogenic signaling pathways was also recently identified for miR-206 in lung squamous cell carcinoma [45]. Therefore, simultaneous inhibition of these RTKs might improve disease treatment. The evidence of miRNA participating in the 
TABLE 2: Alteration of EGFR mutation related microRNAs in lung cancer.

\begin{tabular}{|c|c|c|c|}
\hline MicroRNA(s) & Regulation & Chromosome locus & Reference(s) \\
\hline $\mathrm{miR}-10 \mathrm{~b}$ & Up & $2 \mathrm{q} 31.1$ & {$[30]$} \\
\hline miR-21 & Up & $17 q 23.1$ & {$[26,30]$} \\
\hline $\operatorname{miR}-122$ & Up & $18 \mathrm{q} 21.3$ & {$[34]$} \\
\hline miR-134/487b/655 cluster & Up & $14 \mathrm{q} 32$ & [49] \\
\hline miR-183-3p & Up & $7 \mathrm{p} 32$ & {$[35]$} \\
\hline miR-200b & Up & $1 \mathrm{p} 36.33$ & {$[30]$} \\
\hline $\operatorname{miR}-210$ & Up & 11p15.5 & {$[30]$} \\
\hline miR-30a & Down & $6 \mathrm{q} 13$ & {$[30]$} \\
\hline miR-30b & Down & $8 \mathrm{q} 24.22$ & {$[30,50]$} \\
\hline miR-30c & Down & $1 \mathrm{p} 34.2$ & {$[50]$} \\
\hline $\operatorname{miR}-34 a$ & Down & $1 \mathrm{p} 36.23$ & {$[51]$} \\
\hline $\operatorname{miR}-126$ & Down & $9 q 34.3$ & {$[30]$} \\
\hline miR-145 & Down & $5 q 32-33$ & {$[31]$} \\
\hline $\operatorname{miR}-451$ & Down & $17 q 11.2$ & {$[30]$} \\
\hline
\end{tabular}

TABLE 3: MicroRNAs regulate chemoresistance in lung cancer.

\begin{tabular}{|c|c|c|c|c|}
\hline MicroRNA(s) & Regulation & Agent & Target(s) & Reference(s) \\
\hline let-7 & Down & Erlotinib & Hedgehog & {$[52]$} \\
\hline miR-34 & Down & Gefitinib & c-MET/HGF & [51] \\
\hline miR-103 & Down & Gefitinib & PKC- $\varepsilon$ & [32] \\
\hline $\operatorname{miR}-128 b$ & Down & Gefitinib & EGFR & [15] \\
\hline miR-138-5p & Down & Gefitinib & GPR124 & [53] \\
\hline miR-145 & Down & TKIs & ERK, AKT, OCT4, c-MYC, EGFR, and NUDT1 & {$[31,54]$} \\
\hline miR-146a & Down & TKIs & EGFR and NF- $\kappa \mathrm{B}$ & {$[40]$} \\
\hline miR-147 & Down & Gefitinib & ZEB1 and AKT & {$[55]$} \\
\hline $\operatorname{miR}-200$ & Down & Erlotinib & Hedgehog, MIG6, and TGF $\beta 1$ & {$[52,56,57]$} \\
\hline miR-203 & Down & Gefitinib & SRC & {$[32]$} \\
\hline $\operatorname{miR}-424$ & Down & TKIs & Not applicable & [15] \\
\hline miR-548b & Down & TKIs & CCNB1 & {$[58]$} \\
\hline miR-7 & Up & TKIs & EGFR, RAF1, and IRS-1 & {$[21,59,60]$} \\
\hline miR-21 & Up & Gefitinib & PTEN, MDR1, Bcl-2, and PDCD4 & [33] \\
\hline $\mathrm{miR}-30 \mathrm{~b} / 30 \mathrm{c}$ & Up & Gefitinib & BIM & {$[32]$} \\
\hline $\operatorname{miR}-126$ & Up & Gefitinib & AKT, EGFL7, PI3KR2, ERK, CRK, and VEGF & {$[54]$} \\
\hline miR-134/487b/655 cluster & Up & Gefitinib & MAGI2 & [49] \\
\hline $\operatorname{miR}-221 / 222$ & Up & Gefitinib & APAF-1 & {$[32]$} \\
\hline $\operatorname{miR}-214$ & Up & TKIs & PTEN, MAPK, and p38 & {$[46]$} \\
\hline miR-374a & Up & TKIs & Wnt5a & [58] \\
\hline
\end{tabular}

EGFR/c-MET network in lung cancer thus provides a new clue to overcoming EGFR-TKI resistance in lung cancer [63].

\section{4. miRNAs as Biomarkers for Predicting EGFR-TKI Response in Lung Cancer}

Given the fact that only small portion of patients with lung cancer benefit from a treatment of EGFR-TKIs, the benefits of these agents to patients are ultimately limited by the emergence of drug resistance [64]. Therefore, great efforts have been made to identify new biomarkers for predicting responses to TKI treatment in lung cancer. The ability to alter
EGFR-TKIs responses makes miRNAs as potential predictive biomarkers for EGFR-TKIs in lung cancer treatment, and several miRNAs could be served as biomarkers to predict response to EGFR-TKIs in lung cancer patients have been recently well documented (Table 3 ) $[9,65]$.

In a miRNA profiling analysis with retrospective cohorts consisted of 128 radically resected NSCLC patients (60 were EGFR mutation positive, 68 were negative, and 32 healthy controls), Shen et al. found that the expression of miR21 and miR-10b in radically resected NSCLC patients with EGFR mutation were much higher relative to those without mutation. A Cox proportional-hazards regression analysis 
further demonstrated that a reduced expression of miR-21 was associated with a significant improvement in overall survival in patients treated with gefitinib; that is, a patient who had upregulated miR-21 expression might have poor overall survival, but a better response to gefitinib. This data suggests that miR-21 expression may be an independent predictor of the response to gefitinib in lung cancer [30]. Of interest, an aberrant expression of miR-21 was also significantly correlated with platinum-based chemotherapy resistance in NSCLC patients, and an increased miR-21 expression was associated with the shorter disease-free survival [62].

Other miRNAs that targeting the EGFR signaling pathway, such as miR-128b [15], miR-30b, and miR-30c [32], were also significantly correlated with clinical EGFR-TKI responses in lung cancer, in which $\mathrm{LOH}$ at miR-128b is frequent in NSCLC [15], and the expressions of miR-30b and $\mathrm{miR}-30 \mathrm{c}$ have been reported to be prognostic predictors in NSCLC patients who underwent first line treatment with EGFR-TKIs [50]. Gu et al. retrospectively examined expression of miR-30b and miR-30c in 41 NSCLC samples of patients who used TKIs as first line of therapy. They found that there is a significant correlation of miR-30b and miR-30c levels and the short-term TKI responses, suggesting that miR-30b and miR-30c may be useful in predicting TKI response in NSCLC patients [50]. Another study using lung epithelial cancer cell line model has identified 13 miRNA genes to predict response to EGFR inhibitors, among which the miR-200c was able to target epithelial-to-mesenchymal transition (EMT) transcription factor, zinc finger homeodomain enhancer-binding protein 1 (ZEB1) and altered the sensitivity to erlotinib, and migration in lung cells [56]. The transforming growth factor- $\beta$ (TGF- $\beta$ ) is able to induce EMT in cancers $[49,57]$. A negative regulator of EGFR, mitogeninducible gene 6 (MIG6) is a target of miR-200, the ratio of the expression of MIG6/miR200c was found to be tightly correlated with EMT and resistance to erlotinib in lung cancer in vivo, in which the MIG6 (mRNA)/miR200 ratio was inversely correlated with response to erlotinib, indicating that the ratio of MIG6/miR200 may be a predictive biomarker of the response of lung cancer to EGFR-TKIs [57].

\section{5. miRNAs as Therapeutic Targets for Sensitizing EGFR-TKI-Resistant Lung Cancer}

The emerging role of miRNAs in regulation the EGFR signaling pathway and therapeutic responses to EGFR-TKIs has provided a new avenue for developing novel agents and approaches to resensitize TKI resistance and improve the overall clinical outcomes of TKI-treatment in patients with lung cancer. Clinically, majority of EGFR-TKI resistance is induced by a secondary T790M mutation of EGFR or c-MET. In this regard, a T790M mutation is able to enhance the GTP affinity and block TKI binding to the tyrosine kinase domain of EGFR [29]. Therefore, a strategy by targeting G proteincoupled receptor or c-MET may reverse lung cancer cells to EGFR-TKI resistance. In order to overcome the EGFRTKI resistance in T790M mutant NSCLC treatment, Rai et al. delivered miR-7 expressing plasmid to NSCLC cells and xenografts by liposomal transfection and found that the miR7 could inhibit the growth of both TKI sensitive and resistant NSCLC cells in vitro and in vivo [59]. This finding was supported by a late study using Lewis lung cancer (3LL) cells with a downregulated miR-7; this study demonstrated that a restoration of miR-7 inhibited 3LL cell proliferation, induced cell apoptosis in vitro, and reduced tumorigenicity in vivo by targeting the EGFR signaling pathway [60].

Gao et al. recently identified miR-138-5p was strikingly downregulated, which was inversely correlated with the expression of G protein-coupled receptor 124 (GPR124) in a gefitinib-resistant lung cancer cell line PC9GR. Bioinformatics analysis suggested that the GPR124 was a direct target of miR-138-5p, which was further validated experimentally. Intriguingly, forced expression of miR-138-5p was sufficient to resensitize the PC9GR cells and gefitinib resistant NSCLC H1975 cells to gefitinib, and knockdown of GPR124 with small RNA mimics exhibited similar effects of miR-138-5p, suggesting that the acquired gefitinib resistance was in part attributed by a downregulation of miR-138-5p and that restoration of miR-138-5p level might be a potential therapeutic approach for sensitizing gefitinib resistance in NSCLC [53]. Using a similar approach, Zhou et al. found that an ectopic expression of miR-34a could inhibit cell growth and induce apoptosis in hepatocyte growth factor- (HGF-) induced gefitinib-resistant HCC827GR and PC-9GR lung cancer cells and in HGFinduced gefitinib resistant mouse xenograft model, partly by targeting MET [51]. In this context, an upregulation of HGF has been demonstrated as an important mechanism involved in the AR to EGFR-TKIs by activation of PI3K/Akt pathway through phosphorylation of c-MET [32, 36, 62]. This notion was supported by the evidence of that the total and phosphorylated of c-MET proteins were partially decreased in gefitinib-sensitive HCC827 and PC-9 cells, but the total and phosphorylated status of the downstream PI3K/Akt or ERK signaling pathway was not affected by the transfection of miR-34a. This result indicated that miR-34a had an inhibitory effect on MET rather than its downstream signaling components, which also implies that the tumor suppressive effects of miR-34a alone in gefitinib-sensitive EGFR mutant NSCLC cells might mainly be dependent on mechanisms other than c-MET inhibition. This was different from in the gefitinib-resistant HCC827GR and PC-9GR cells, in which a combination of miR-34a and gefitinib could efficiently induced cell death and apoptosis with an inhibition of the phosphorylation of c-MET, EGFR, Akt, and ERK [51]. Such a synergistic effect between the miR-34a and EGFRTKIs was also reported in study of a combination of erlotinib and miR-34a in NSCLC cells with primary and acquired erlotinib resistance, in which a strong synergistic interaction between the erlotinib and miR-34a mimics was observed [51]. These studies clearly suggest that a synergistic strategy using miRNAs and TKIs in a combination may effectively reverse the EGFR-TKI resistance in lung cancer treatment.

Both experimental and preclinical studies have demonstrated that persistent activation of PI3K/Akt and/or Ras/Erk pathways is associated with EGFR-TKI resistance in NSCLC, in which they play pivotal roles in TKI sensitivity [66]. 
Indeed, the overexpression of miR-21 led to a significant decrease of gefitinib sensitivity in PC9 lung cancer cells through a mechanism by inhibiting PTEN expression and activating the Akt/Erk signaling pathway, while knockdown of miR-21 dramatically reversed gefitinib sensitivity in PC9GR cells by upregulating PTEN and inactivating the Akt/Erk pathway, suggesting modulation of miR-21/PTEN expression may be a promising strategy for resensitizing EGFR-TKI resistance in NSCLC [33].

Apart from targeting the PI3K/Akt/Ras/Erk pathway, EMT is involved in the AR to therapy, which is often activated during the progression of lung cancer $[52,56$, 57, 67]. Several lines of evidence have demonstrated that miRNAs are involved in MET and reverse EGFR-TKI resistance in NSCLC [55, 57, 67]. For instances, miR-147 was downregulated in NSCLC, and overexpression of miR-147 could induce the MET of lung cancer cells, sequentially resensitize the resistance to EGFR-TKIs by inhibiting the Akt signaling pathway, and the MET phenotype of lung cancer cells could be attenuated by TGF- $\beta$ [55]. Exposure NSCLC cells with EMT to TGF- $\beta 1$ was found to significantly induce miR-134/487b/655 cluster by targeting membraneassociated guanylate kinase, WW domain- and PDZ domaincontaining protein 2 (MAGI2), a scaffold protein required for PTEN. Ectopic expression of miR-134 and miR-487b enhanced the EMT potential and the drug resistance to gefitinib of the cells, whereas reduction of the transcripts of these miRNAs led to an inhibition of EMT process and a restoration of drug sensitivity of TGF- $\beta 1$-induced resistance to gefitinib, implying that the miR-134/miR-487b/miR-655 cluster may be a novel therapeutic target in patients with advanced lung adenocarcinoma [49]. By modulating EMTregulating miRNAs, Ahmad et al. demonstrated that both specific siRNA to the Hedgehog $(\mathrm{HH})$ signaling pathway and GDC-0449 (a small molecule antagonist of G protein coupled receptor smoothened in the $\mathrm{HH}$ pathway) were able to resensitize TGF- $\beta 1$-induced erlotinib resistant A549 (A549M) cells with an upregulation of miR-200b and let7c. Ectopic expression of these miRNAs also led to diminish the erlotinib resistance of A549M cells [52]. In another study, Cufí et al. discovered that flavonolignan silibinin could suppress the EMT-driven erlotinib resistance by restoring a high miR-21/low miR-200c signature in EGFR-mutant NSCLC xenografts [67]. A combination of erlotinib and silibinin led a completely abrogate tumor growth in the NSCLC xenograft model. Mechanistically, the silibinin could fully restore the EMT-related high miR-21/low miR-200c signature and inhibit the expression of mesenchymal markers snail family zinc finger 1 (SNAI1), ZEB, and N-cadherin in erlotinib-refractory tumors. In addition, the silibinin was sufficient to fully activate a reciprocal c-MET in erlotinibrefractory cells [67].

In addition to T790M and c-MET amplification, Axl kinase is found to be upregulated in humans with acquired resistance to EGFR-TKI, and the involvement of Axl kinase in acquired resistance of NSCLC to RGFR-TKIs gefitinib or erlotinib has been reported $[38,58,68]$. In order to interrogate the role of miRNAs in the Axl-mediated acquired TKIs resistance in lung cancer, Wang et al. identified a panel of Axl kinase-altered miRNAs in lung cancer cells and experimentally validated that the Axl-induced miR-374a and miR-548b play a crucial role in cell cycle arrest, gefitinibinduced apoptosis, and EMT of gefitinib-resistant lung cancer cells by targeting Wnt5a and CCNB1 genes, respectively. Clinically, a high expression of Axl and miR-374a and low abundance of miR-548b are associated with poor disease-free survival. These observations suggest a promising strategy by targeting miRNAs to reverse gefitinib resistance in NSCLC with high expression of Axl [58, 68].

\section{Perspectives and Challenges}

As a class of regulators at the posttranscription level, miRNAs display different expression patterns in various types of cancer, in which some miRNAs are dysregulated and they play crucial roles in the initiation, progression, and therapeutic responses of cancer. The involvement of miRNAs in the mutant status of EGFR and the emerging role of these molecules in the regulation of the EGFR signaling pathway and drug resistance to anti-EGFR agents have made miRNAs potential biomarkers for the diagnosis and prognosis of lung cancer, as well as potential predictive markers for the therapeutic outcome using anti-EGFR agents or regimens. In a therapeutic standpoint, in addition to restore the functions of tumor suppressor genes or inhibiting oncogenes by targeting miRNAs in anticancer therapy, the modulation of miRNA profiles is also a plausible therapeutic strategies to resensitizing chemoresistance in cancer treatment. In terms of anti-EGFR therapy, an intervention of specific miRNAs that involved in the EGFR signaling pathway and/or EGFRTKI resistance has shown a promising effect to reverse the resistance of lung cancer cells to anti-EGFR therapy by enhancing the sensitivity of tumor cells to chemotherapy or inhibiting cancer cell stemness.

Although recent studies in lung cancer and miRNAs have significantly extended our understanding of the EGFR signaling pathway and its involvement in the pathogenic processes of lung cancer, our understanding of the underlying mechanisms that integrate the activity of this pathway remains fragmentary. Therefore, intensively exploring the regulatory roles of miRNAs in this pathway may contribute to the possible implementation of miRNAs as predictive and prognostic biomarkers $[69,70]$. Particularly, the application of miRNAs as predictive biomarkers may also be beneficial for predicting therapeutic response to anti-EGFR agents in advanced lung cancer patients and lead to a higher level of personalized therapy. However, challenges for the development of miRNA in therapy remain to be addressed; these include tissue specific delivery, potential off-target effects, and safety. An improvement of the specificity of miRNAs and the development of efficient systemic delivery approaches will facilitate the use of miRNAs for the treatment of patients with lung cancer.

\section{Abbreviations}

AKT: $\quad$ Protein kinase B

APAF-1: Apoptotic peptidase activating factor 1 


\begin{tabular}{|c|c|}
\hline Axl: & Anexelekto \\
\hline BIM: & BCL2-like 11 \\
\hline $\begin{array}{l}\text { CCNB1: } \\
\text { CCND1: }\end{array}$ & $\begin{array}{l}\text { Cyclin B1 } \\
\text { Cyclin D1 }\end{array}$ \\
\hline MET: & $\begin{array}{l}\text { The transmembrane tyrosine kinase cell } \\
\text { surface receptor for HGF }\end{array}$ \\
\hline -Myc: & $\begin{array}{l}\text { A regulator gene that codes for a } \\
\text { transcription factor }\end{array}$ \\
\hline CRK: & CT10 regulator of kinase (Crk) \\
\hline EGFR: & Epidermal growth factor receptor \\
\hline MT: & Epithelial to mesenchymal transition \\
\hline RF: & Ets2 transcriptional repression factor \\
\hline RK: & Extracellular signal-regulated kinase \\
\hline PR124: & G protein-coupled receptor 124 \\
\hline GF: & Hepatocyte growth factor \\
\hline HH: & Hedgehog \\
\hline IGF1R: & Insulin-like growth factor 1 receptor \\
\hline RS-1: & Insulin receptor substrate-1 \\
\hline K-Ras: & $\begin{array}{l}\text { Kirsten rat sarcoma viral oncogene } \\
\text { homolog }\end{array}$ \\
\hline LOH: & Loss of heterozygosity \\
\hline AGI?. & $\begin{array}{l}\text { Membrane-associated guanylate kinase, } \\
\text { WW, and PDZ domain-containing protein } \\
2\end{array}$ \\
\hline MAPK: & Mitogen-activated protein kinase \\
\hline IDR-1: & Multidrug resistan \\
\hline MET: & Mesenchymal to epithelial transition \\
\hline MIG6: & Mitogen-inducible gene 6 \\
\hline$N F-\kappa B:$ & Nuclear factor kappa beta \\
\hline NSCLC: & Non-small-cell lung cancer \\
\hline NUDT1: & $\begin{array}{l}\text { Nucleoside diphosphate linked moiety } \\
\text { X-type motif } 1\end{array}$ \\
\hline PDCD4: & Programmed cell death protein 4 \\
\hline PDZ dom & $\begin{array}{l}\text { An interaction motif that recognizes and } \\
\text { binds the C-terminal peptides of target } \\
\text { proteins }\end{array}$ \\
\hline PI3K: & Phosphatidylinositol-3-kinase \\
\hline PKC- $\varepsilon$ : & Protein kinase $\mathrm{C}$ varepsilon \\
\hline PTEN: & Phosphatase and tensin homolog \\
\hline PTPN9: & $\begin{array}{l}\text { Phosphatases tyrosine-protein phosphatase } \\
\text { nonreceptor type } 9\end{array}$ \\
\hline PTPRF: & $\begin{array}{l}\text { Receptor-type tyrosine-protein } \\
\text { phosphatase F }\end{array}$ \\
\hline RAF-1: & $\begin{array}{l}\text { Proto-oncogene serine/threonine protein } \\
\text { kinase }\end{array}$ \\
\hline RAS: & Rat sarcoma viral oncogene homolog \\
\hline RTK: & Receptor of tyrosine kinase \\
\hline SIRT1: & Sirtuin 1 \\
\hline SNAII: & Snail family zin \\
\hline SRC: & Sarcoma viral oncogene homolog \\
\hline TGF $\beta 1:$ & Transforming growth factor beta 1 \\
\hline TGF $\beta$ R1: & Transforming growth factor beta receptor 1 \\
\hline VEGF: & Vascular endothelial to growth factor \\
\hline Wnt: & $\begin{array}{l}\text { Wingless-type MMTV integration site } \\
\text { family }\end{array}$ \\
\hline mai & $\begin{array}{l}\text { A short conserved protein domain mediat } \\
\text { interactions with protein ligands }\end{array}$ \\
\hline ZEB & $\begin{array}{l}\text { Zinc finger homeodomain } \\
\text { enhancer-binding protein. }\end{array}$ \\
\hline
\end{tabular}

\section{Conflict of Interests}

The authors declare they have no conflict of interests.

\section{Authors' Contribution}

Fei Han and Jinxi He contributed equally to this paper.

\section{Acknowledgment}

This work was supported by grants from the National Natural Science Foundation of China (nos. 31172278, 31472191, and 30960176).

\section{References}

[1] A. Jemal, R. Siegel, J. Xu, and E. Ward, “Cancer statistics, 2010," CA: A Cancer Journal for Clinicians, vol. 60, no. 5, pp. 277-300, 2010.

[2] Y. Wang, G. Deng, X. Liu, and W. C. Cho, "Monoclonal antibodies in lung cancer," Expert Opinion on Biological Therapy, vol.13, no. 2, pp. 209-226, 2013.

[3] J. E. Larsen, T. Cascone, D. E. Gerber, J. V. Heymach, and J. D. Minna, "Targeted therapies for lung cancer: clinical experience and novel agents," Cancer Journal, vol. 17, no. 6, pp. 512-527, 2011.

[4] R. S. Heist and D. Christiani, "EGFR-targeted therapies in lung cancer: predictors of response and toxicity," Pharmacogenomics, vol. 10, no. 1, pp. 59-68, 2009.

[5] T. Yoshida, G. Zhang, and E. B. Haura, "Targeting epidermal growth factor receptor: central signaling kinase in lung cancer," Biochemical Pharmacology, vol. 80, no. 5, pp. 613-623, 2010.

[6] P. A. Jänne, J. A. Engelman, and B. E. Johnson, "Epidermal growth factor receptor mutations in non-small-cell lung cancer: implications for treatment and tumor biology," Journal of Clinical Oncology, vol. 23, no. 14, pp. 3227-3234, 2005.

[7] R. Perez, T. Crombet, J. de Leon, and E. Moreno, "A view on EGFR-targeted therapies from the oncogene-addiction perspective," Frontiers in Pharmacology, vol. 4, article 53, 2013.

[8] J. Remon, T. Morán, M. Majem et al., "Acquired resistance to epidermal growth factor receptor tyrosine kinase inhibitors in EGFR-mutant non-small cell lung cancer: a new era begins," Cancer Treatment Reviews, vol. 40, no. 1, pp. 93-101, 2014.

[9] H. A. Yu, G. J. Riely, and C. M. Lovly, “Therapeutic strategies utilized in the setting of acquired resistance to EGFR tyrosine kinase inhibitors," Clinical Cancer Research, vol. 20, no. 23, pp. 5898-5907, 2014.

[10] Y. Lin, X. Wang, and H. Jin, "EGFR-TKI resistance in NSCLC patients: mechanisms and strategies," The American Journal of Cancer Research, vol. 4, no. 5, pp. 411-435, 2014.

[11] M. Inui, G. Martello, and S. Piccolo, "MicroRNA control of signal transduction," Nature Reviews Molecular Cell Biology, vol. 11, no. 4, pp. 252-263, 2010.

[12] I. Hoshino and H. Matsubara, "MicroRNAs in cancer diagnosis and therapy: from bench to bedside," Surgery Today, vol. 43, no. 5, pp. 467-478, 2013.

[13] F. Wang, L. W. Chan, H. K. Law et al., "Exploring microRNAmediated alteration of EGFR signaling pathway in non-small cell lung cancer using an mRNA:miRNA regression model supported by target prediction databases," Genomics, vol. 104, no. 6, pp. 504-511, 2014. 
[14] D. N. Hayes and H. L. McLeod, "EGFR regulation by microRNA in lung cancer: a rose by any other name ... is an increasingly complicated rose," Annals of Oncology, vol. 19, no. 6, pp. 10361037, 2008.

[15] G. J. Weiss, L. T. Bemis, E. Nakajima et al., "EGFR regulation by microRNA in lung cancer: correlation with clinical response and survival to gefitinib and EGFR expression in cell lines," Annals of Oncology, vol. 19, no. 6, pp. 1053-1059, 2008.

[16] G. G. Gomez, J. Wykosky, C. Zanca, F. B. Furnari, and W. K. Cavenee, "Therapeutic resistance in cancer: microRNA regulation of EGFR signaling networks," Cancer Biology and Medicine, vol. 10, no. 4, pp. 192-205, 2013.

[17] R. M. Mader, S. Foerster, N. Sarin et al., "NSCLC cells adapted to EGFR inhibition accumulate EGFR interacting proteins and down-regulate microRNA related to epithelial-mesenchymal transition," International Journal of Clinical Pharmacology and Therapertics, vol. 52, no. 1, pp. 92-94, 2014.

[18] L. W. C. Chan, F. F. Wang, and W. C. S. Cho, "Genomic sequence analysis of EGFR regulation by microRNAs in lung cancer," Current Topics in Medicinal Chemistry, vol. 12, no. 8, pp. 920 926, 2012.

[19] B. Kefas, J. Godlewski, L. Comeau et al., "microRNA-7 inhibits the epidermal growth factor receptor and the Akt pathway and is down-regulated in glioblastoma," Cancer Research, vol. 68, no. 10, pp. 3566-3572, 2008.

[20] R. J. Webster, K. M. Giles, K. J. Price, P. M. Zhang, J. S. Mattick, and P. J. Leedman, "Regulation of epidermal growth factor receptor signaling in human cancer cells by MicroRNA-7," The Journal of Biological Chemistry, vol. 284, no. 9, pp. 5731-5741, 2009.

[21] Y.-T. Chou, H.-H. Lin, Y.-C. Lien et al., "EGFR promotes lung tumorigenesis by activating miR-7 through a Ras/ERK/Myc pathway that targets the Ets2 transcriptional repressor ERF," Cancer Research, vol. 70, no. 21, pp. 8822-8831, 2010.

[22] M. M. Bjaanaes, A. R. Halvorsen, S. Solberg et al., "Unique microRNA-profiles in EGFR-mutated lung adenocarcinomas," International Journal of Cancer, vol. 135, no. 8, pp. 1812-1821, 2014.

[23] S. Dacic, L. Kelly, Y. Shuai, and M. N. Nikiforova, "miRNA expression profiling of lung adenocarcinomas: correlation with mutational status," Modern Pathology, vol. 23, no. 12, pp. 15771582, 2010.

[24] G. Yamaguchi, M. Takanashi, M. Tanaka et al., "Isolation of miRNAs that target EGFR mRNA in human lung cancer," Biochemical and Biophysical Research Communications, vol. 420, no. 2, pp. 411-416, 2012.

[25] L.-K. Wang, T.-H. Hsiao, T.-M. Hong et al., "MicroRNA-133a suppresses multiple oncogenic membrane receptors and cell invasion in non-small cell lung carcinoma," PLoS ONE, vol. 9, no. 5, Article ID e96765, 2014.

[26] M. Seike, A. Goto, T. Okano et al., "miR-21 is an EGFR-regulated anti-apoptotic factor in lung cancer in never-smokers," Proceedings of the National Academy of Sciences of the United States of America, vol. 106, no. 29, pp. 12085-12090, 2009.

[27] Y. H. Guo, C. Zhang, J. Shi et al., "Abnormal activation of the EGFR signaling pathway mediates the downregulation of miR145 through the ERK1/2 in non-small cell lung cancer," Oncology Reports, vol. 31, no. 4, pp. 1940-1946, 2014.

[28] Y.-L. Wu, C. Zhou, C.-P. Hu et al., "Afatinib versus cisplatin plus gemcitabine for first-line treatment of Asian patients with advanced non-small-cell lung cancer harbouring EGFR mutations (LUX-Lung 6): an open-label, randomised phase 3 trial," The Lancet Oncology, vol. 15, no. 2, pp. 213-222, 2014.

[29] K.-Y. Su, H.-Y. Chen, K.-C. Li et al., "Pretreatment Epidermal Growth Factor Receptor (EGFR) T790M mutation predicts shorter EGFR tyrosine kinase inhibitor response duration in patients with non-small-cell lung cancer," Journal of Clinical Oncology, vol. 30, no. 4, pp. 433-440, 2012.

[30] Y. Shen, D. Tang, R. Yao et al., "MicroRNA expression profiles associated with survival, disease progression, and response to gefitinib in completely resected non-small-cell lung cancer with EGFR mutation," Medical Oncology, vol. 30, no. 4, article 750, 2013.

[31] W. C. S. Cho, A. S. C. Chow, and J. S. K. Au, "MiR-145 inhibits cell proliferation of human lung adenocarcinoma by targeting EGFR and NUDT1," RNA Biology, vol. 8, no. 1, pp. 125-131, 2011.

[32] M. Garofalo, G. Romano, G. Di Leva et al., "EGFR and MET receptor tyrosine kinase-altered microRNA expression induces tumorigenesis and gefitinib resistance in lung cancers," Nature Medicine, vol. 18, no. 1, pp. 74-82, 2012.

[33] B. Li, S. Ren, X. Li et al., "MiR-21 overexpression is associated with acquired resistance of EGFR-TKI in non-small cell lung cancer," Lung Cancer, vol. 83, no. 2, pp. 146-153, 2014.

[34] H. Zhang, Y. Su, F. Xu, J. Kong, H. Yu, and B. Qian, “Circulating microRNAs in relation to EGFR status and survival of lung adenocarcinoma in female non-smokers," PLOS ONE, vol. 8, no. 11, Article ID e81408, 2013.

[35] F. Xu, H. Zhang, Y. Su, J. Kong, H. Yu, and B. Qian, "Upregulation of microRNA-183-3p is a potent prognostic marker for lung adenocarcinoma of female non-smokers," Clinical and Translational Oncology, vol. 16, no. 11, pp. 980-985, 2014.

[36] M. Acunzo, G. Romano, D. Palmieri et al., "Cross-talk between MET and EGFR in non-small cell lung cancer involves miR-27a and Sprouty2," Proceedings of the National Academy of Sciences of the United States of America, vol. 110, no. 21, pp. 8573-8578, 2013.

[37] W. Wang, B. Cheng, L. Miao, Y. Me, and M. Wu, "Mutant p53$\mathrm{R} 273 \mathrm{H}$ gains new function in sustained activation of EGFR signaling via suppressing miR-27a expression," Cell Death and Disease, vol. 4, no. 4, article e574, 2013.

[38] G. Mudduluru, P. Ceppi, R. Kumarswamy, G. V. Scagliotti, M. Papotti, and H. Allgayer, "Regulation of Axl receptor tyrosine kinase expression by miR-34a and miR-199a/b in solid cancer," Oncogene, vol. 30, no. 25, pp. 2888-2899, 2011.

[39] L. Liu, X. Shao, W. Gao et al., "MicroRNA-133b inhibits the growth of non-small-cell lung cancer by targeting the epidermal growth factor receptor," The FEBS Journal, vol. 279, no. 20, pp. 3800-3812, 2012.

[40] G. Chen, I. A. Umelo, S. Lv et al., "miR-146a inhibits cell growth, cell migration and induces apoptosis in non-small cell lung cancer cells," PLoS ONE, vol. 8, no. 3, Article ID e60317, 2013.

[41] B. Xu, N. Wang, X. Wang et al., "miR-146a suppresses tumor growth and progression by targeting EGFR pathway and in a p-ERK-dependent manner in castration-resistant prostate cancer," Prostate, vol. 72, no. 11, pp. 1171-1178, 2012.

[42] M. Katakowski, X. Zheng, F. Jiang, T. Rogers, A. Szalad, and M. Chopp, "MiR-146b-5p suppresses EGFR expression and reduces In Vitro migration and invasion of glioma," Cancer Investigation, vol. 28, no. 10, pp. 1024-1030, 2010.

[43] L. Adam, M. Zhong, W. Choi et al., "miR-200 expression regulates epithelial-to-mesenchymal transition in bladder cancer cells and reverses resistance to epidermal growth factor receptor 
therapy," Clinical Cancer Research, vol. 15, no. 16, pp. 5060-5072, 2009.

[44] Z. Zhang, Z.-B. Liu, W.-M. Ren, X.-G. Ye, and Y.-Y. Zhang, “The miR-200 family regulates the epithelial-mesenchymal transition induced by EGF/EGFR in anaplastic thyroid cancer cells," International Journal of Molecular Medicine, vol. 30, no. 4, pp. 856-862, 2012.

[45] H. Mataki, N. Seki, T. Chiyomaru et al., "Tumor-suppressive microRNA-206 as a dual inhibitor of MET and EGFR oncogenic signaling in lung squamous cell carcinoma," International Journal of Oncology, vol. 46, no. 3, pp. 1039-1050, 2015.

[46] X. Zhou, Y. Ren, L. Moore et al., "Downregulation of miR-21 inhibits EGFR pathway and suppresses the growth of human glioblastoma cells independent of PTEN status," Laboratory Investigation, vol. 90, no. 2, pp. 144-155, 2010.

[47] W. W. Du, L. Fang, M. Li et al., "MicroRNA miR-24 enhances tumor invasion and metastasis by targeting PTPN9 and PTPRF to promote EGF signaling," Journal of Cell Science, vol. 126, no. 6, pp. 1440-1453, 2013.

[48] H. Salim, N. S. Akbar, D. Zong et al., "MiRNA-214 modulates radiotherapy response of non-small cell lung cancer cells through regulation of p38MAPK, apoptosis and senescence," British Journal of Cancer, vol. 107, no. 8, pp. 1361-1373, 2012.

[49] K. Kitamura, M. Seike, T. Okano et al., "MiR-134/487b/655 cluster regulates TGF- $\beta$-induced epithelial- mesenchymal transition and drug resistance to gefitinib by targeting MAGI2 in lung adenocarcinoma cells," Molecular Cancer Therapeutics, vol. 13, no. 2, pp. 444-453, 2014.

[50] Y.-F. Gu, H. Zhang, D. Su et al., "miR-30b and miR-30c expression predicted response to tyrosine kinase inhibitors as first line treatment in non-small cell lung cancer," Chinese Medical Journal, vol. 126, no. 23, pp. 4435-4439, 2013.

[51] J. Zhou, X. Chen, J. Zhao, Z. Bao, P. Zhang, and Z. Liu, "MicroRNA-34a overcomes HGF-mediated gefitinib resistance in EGFR mutant lung cancer cells partly by targeting MET," Cancer Letters, vol. 351, no. 2, pp. 265-271, 2014.

[52] A. Ahmad, M. Y. Maitah, K. R. Ginnebaugh et al., "Inhibition of Hedgehog signaling sensitizes NSCLC cells to standard therapies through modulation of EMT-regulating miRNAs," Journal of Hematology and Oncology, vol. 6, no. 1, article 77, 2013.

[53] Y. Gao, X. Fan, W. Li, W. Ping, Y. Deng, and X. Fu, "MiR-138$5 p$ reverses gefitinib resistance in non-small cell lung cancer cells via negatively regulating G protein-coupled receptor 124," Biochemical and Biophysical Research Communications, vol. 446, no. 1, pp. 179-186, 2014.

[54] M. Zhong, X. Ma, C. Sun, and L. Chen, "MicroRNAs reduce tumor growth and contribute to enhance cytotoxicity induced by gefitinib in non-small cell lung cancer," Chemico-Biological Interactions, vol. 184, no. 3, pp. 431-438, 2010.

[55] C. G. Lee, S. McCarthy, M. Gruidl, C. Timme, and T. J. Yeatman, "MicroRNA-147 induces a mesenchymal-to-epithelial transition (MET) and reverses EGFR inhibitor resistance," PLoS ONE, vol. 9, no. 1, Article ID e84597, 2014.

[56] J. L. Bryant, J. Britson, J. M. Balko et al., "A microRNA gene expression signature predicts response to erlotinib in epithelial cancer cell lines and targets EMT," British Journal of Cancer, vol. 106, no. 1, pp. 148-156, 2012.

[57] E. Izumchenko, X. Chang, C. Michailidi et al., "The TGFbetamiR200-MIG6 pathway orchestrates the EMT-associated kinase switch that induces resistance to EGFR inhibitors," Cancer Research, vol. 74, no. 14, pp. 3995-4005, 2014.
[58] Y. Wang, H. Xia, Z. Zhuang, L. Miao, X. Chen, and H. Cai, "Axl-altered microRNAs regulate tumorigenicity and gefitinib resistance in lung cancer," Cell Death \& Disease, vol. 5, no. 5, Article ID e1227, 2014.

[59] K. Rai, N. Takigawa, S. Ito et al., "Liposomal delivery of MicroRNA-7-expressing plasmid overcomes epidermal growth factor receptor tyrosine kinase inhibitor-resistance in lung cancer cells," Molecular Cancer Therapeutics, vol. 10, no. 9, pp. 1720-1727, 2011.

[60] J. Li, Y. Zheng, G. Sun, and S. Xiong, "Restoration of miR7 expression suppresses the growth of Lewis lung cancer cells by modulating epidermal growth factor receptor signaling," Oncology Reports, vol. 32, no. 6, pp. 2511-2516, 2014.

[61] H.-S. Jeon, Y. H. Lee, S. Y. Lee et al., "A common polymorphism in pre-microRNA-146a is associated with lung cancer risk in a Korean population," Gene, vol. 534, no. 1, pp. 66-71, 2014.

[62] J. A. Engelman, K. Zejnullahu, T. Mitsudomi et al., "MET amplification leads to gefitinib resistance in lung cancer by activating ERBB3 signaling," Science, vol. 316, no. 5827, pp. 10391043, 2007.

[63] M. Garofalo and C. M. Croce, "MicroRNAs: master regulators as potential therapeutics in cancer," Annual Review of Pharmacology and Toxicology, vol. 51, pp. 25-43, 2011.

[64] K. Politi, P.-D. Fan, R. Shen, M. Zakowski, and H. Varmus, "Erlotinib resistance in mouse models of epidermal growth factor receptor-induced lung adenocarcinoma," Disease Models \& Mechanisms, vol. 3, no. 1-2, pp. 111-119, 2010.

[65] V. Del Vescovo, M. Grasso, M. Barbareschi, and M. A. Denti, "MicroRNAs as lung cancer biomarkers," World Journal of Clinical Oncology, vol. 5, no. 4, pp. 604-620, 2014.

[66] I. S. Donev, W. Wang, T. Yamada et al., "Transient PI3K inhibition induces apoptosis and overcomes HGF-mediated resistance to EGFR-TKIs in EGFR mutant lung cancer," Clinical Cancer Research, vol. 17, no. 8, pp. 2260-2269, 2011.

[67] S. Cufí, R. Bonavia, A. Vazquez-Martin et al., "Silibinin suppresses EMT-driven erlotinib resistance by reversing the high miR-21/low miR-200c signature in vivo," Scientific Reports, vol. 3, article 2459, 2013.

[68] Z. Zhang, J. C. Lee, L. Lin et al., "Activation of the AXL kinase causes resistance to EGFR-targeted therapy in lung cancer," Nature Genetics, vol. 44, no. 8, pp. 852-860, 2012.

[69] W. C. S. Cho, "Targeting signaling pathways in lung cancer therapy," Expert Opinion on Therapeutic Targets, vol. 17, no. 2, pp. 107-111, 2013.

[70] W. C. S. Cho, "MicroRNAs: potential biomarkers for cancer diagnosis, prognosis and targets for therapy," International Journal of Biochemistry and Cell Biology, vol. 42, no. 8, pp. 12731281, 2010. 

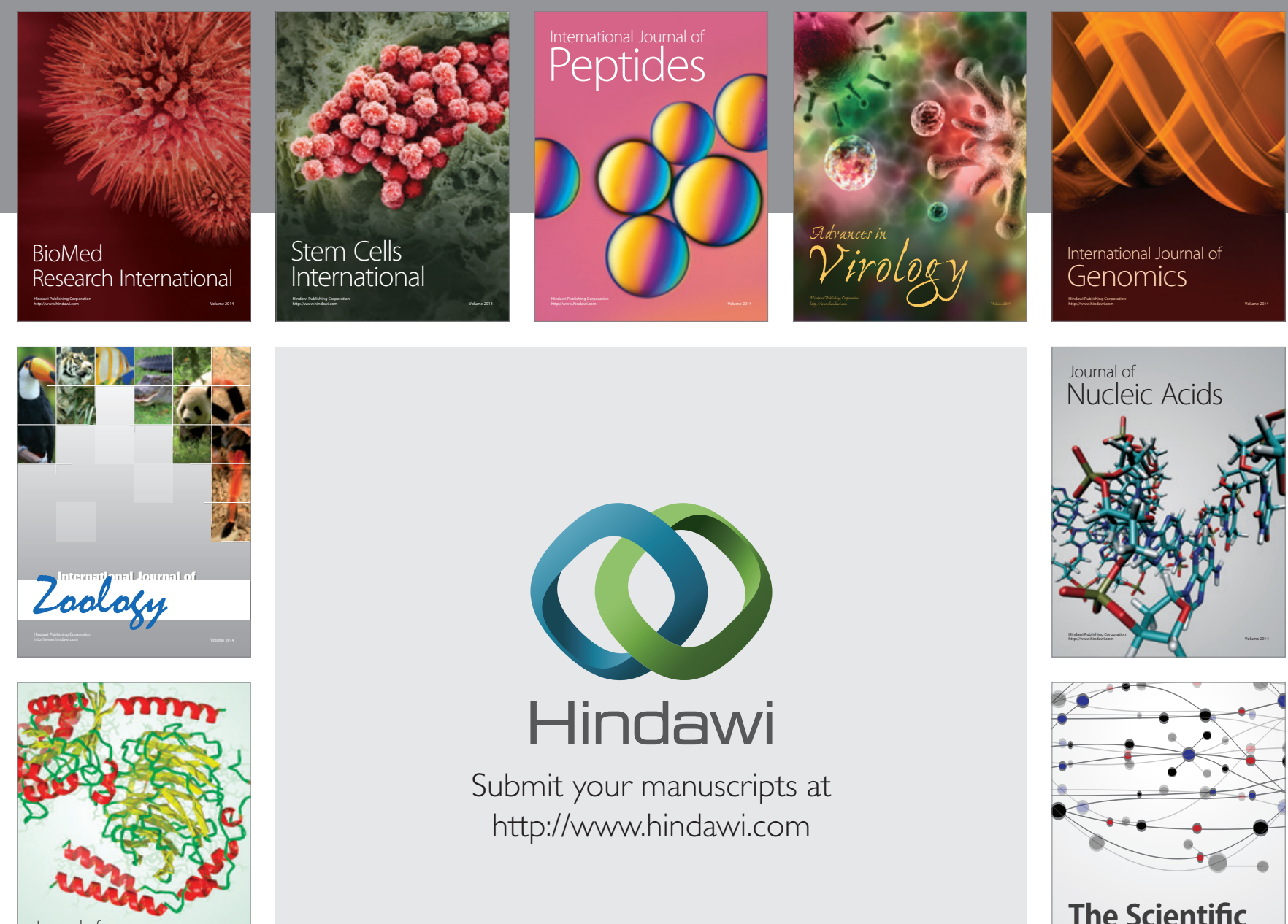

Submit your manuscripts at

http://www.hindawi.com

Journal of
Signal Transduction
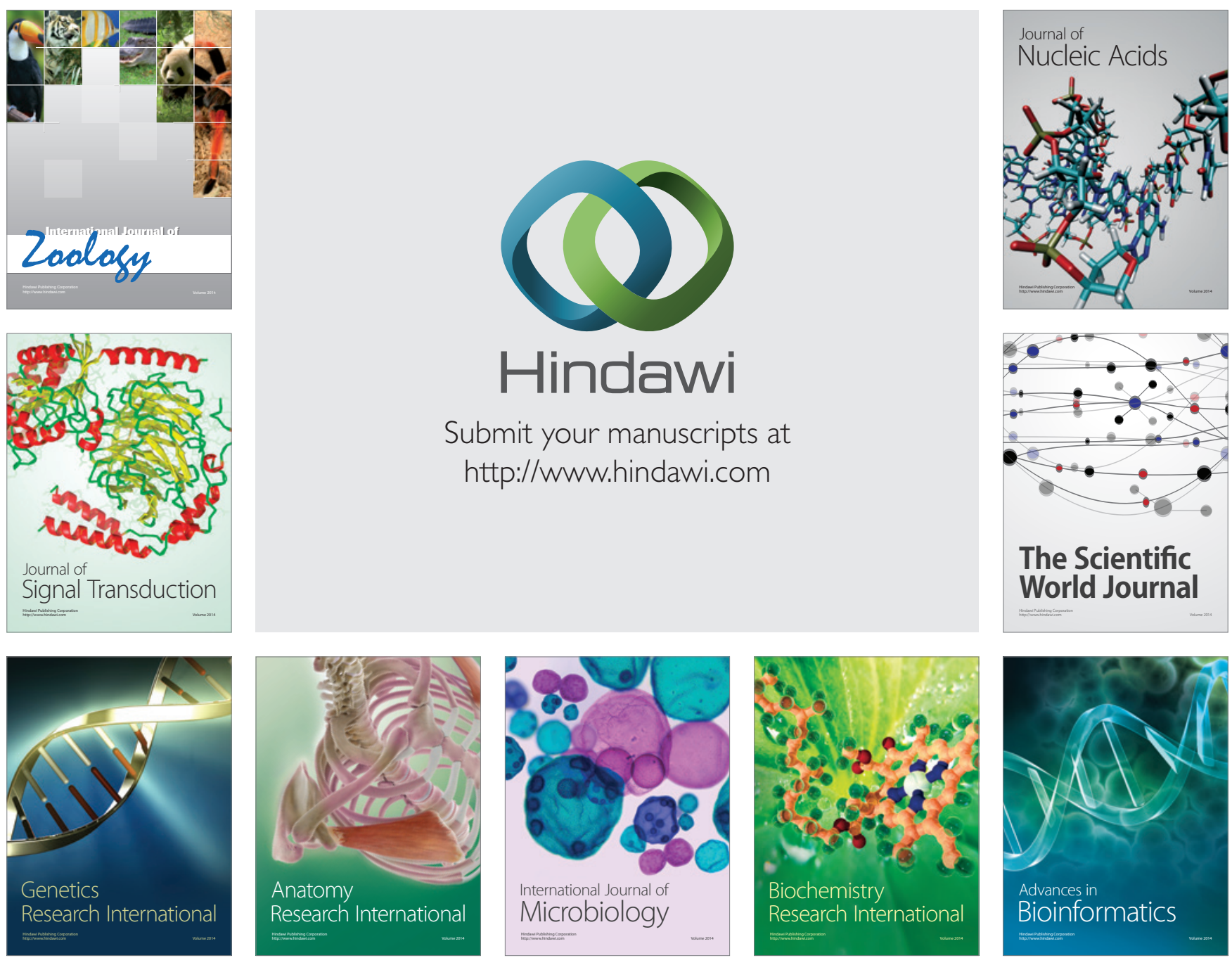

The Scientific World Journal
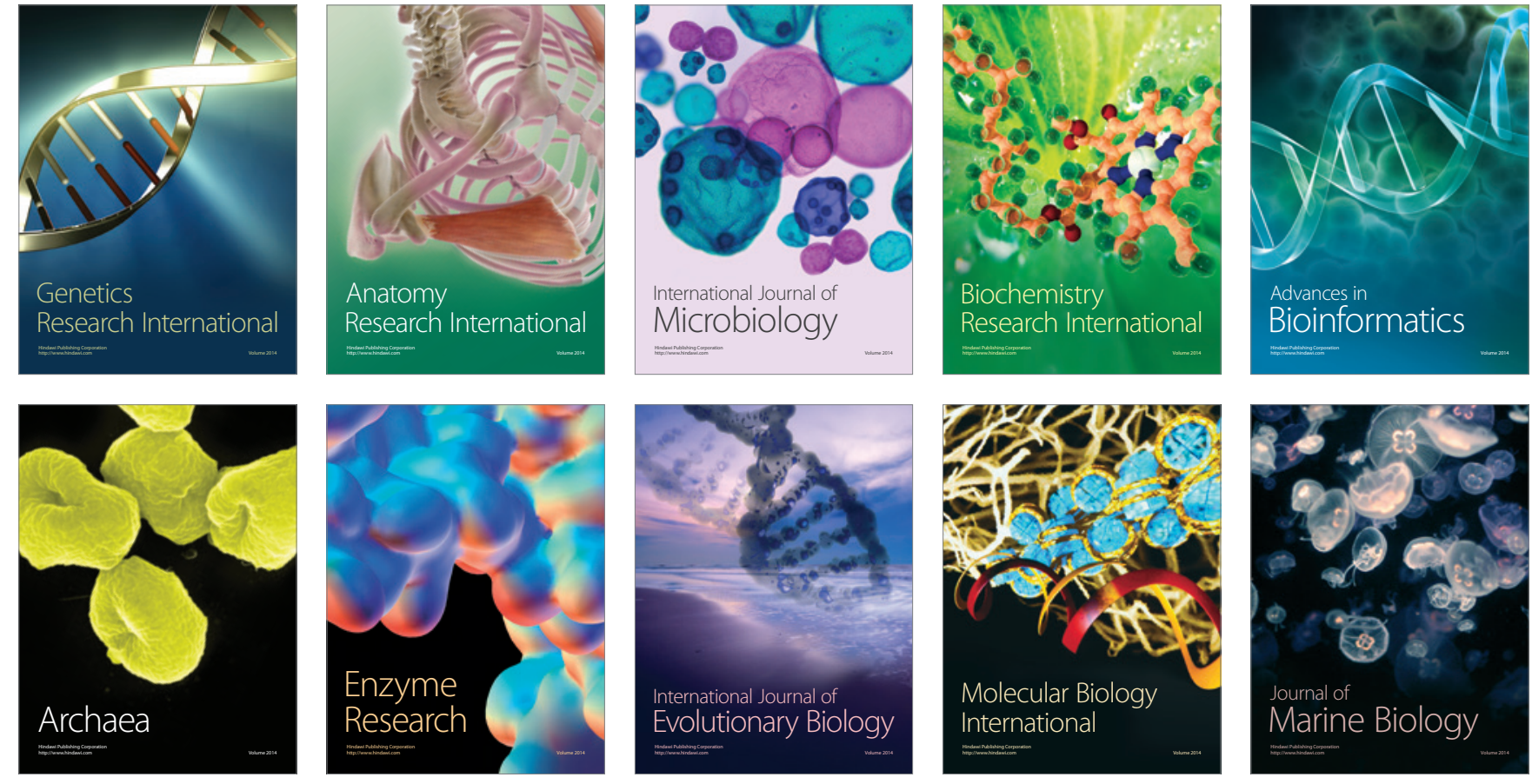